Novel Amalgaviruses of Rubber Dandelion

\title{
Title: Molecular Identification and Characterization of Two Rubber Dandelion
}

\section{Amalgaviruses}

Humberto Debat ${ }^{1 *}$, Zinan $\mathrm{Luo}^{2}$, Brian J. Iaffaldano ${ }^{2}$, Xiaofeng Zhuang ${ }^{2}$ and Katrina Cornish ${ }^{2}$

${ }^{1}$ Instituto de Patología Vegetal, Centro de Investigaciones Agropecuarias, Instituto Nacional de

Tecnología Agropecuaria (IPAVE-CIAP-INTA), 11 de setiembre 4755, Córdoba, Argentina, X5020ICA.

${ }^{2}$ Department of Horticulture and Crop Science, The Ohio State University, Ohio Agricultural Research and Development Center, Williams Hall, 1680 Madison Avenue, Wooster, OH 44691.

* Corresponding author: Humberto Debat, debat.humberto@inta.gob.ar, Tel: +54 9351 4973636, Fax:

+5493514974330 
Novel Amalgaviruses of Rubber Dandelion

\section{Summary}

The Amalgaviridae family is composed of persistent viruses that share the genome architecture of Totiviridae and gene evolutionary resemblance to Partitiviridae. A single Amalgavirus genus has been assigned to this family, presenting only four recognized species, corresponding to plant infecting viruses with dsRNA monopartite genomes of ca. $3.4 \mathrm{~kb}$. Here, we present the genomic identification and characterization of two novel viruses detected in rubber dandelion (Taraxacum kok-saghyz). The sequenced isolates presented genomes of 3,409 and 3,413 nt long, including two partially overlapping ORFs encoding a putative coat protein and an RNA-dependent RNA polymerase (RdRP). Phylogenetic insights based on the detected virus sequences suggest them to be members of two new species within the Amalgavirus genus. Multiple independent RNAseq data suggest that the identified viruses have a dynamic distribution and low relative RNA levels in infected plants. Virus presence was not associated with any apparent symptoms on the plant hosts. We propose the names rubber dandelion latent virus $1 \&$ 2 to the detected amalgaviruses; the first viruses to be associated to this emergent and sustainable natural rubber crop.

\section{Keywords}

Amalgavirus, Rubber dandelion, dsRNA virus, deep sequencing, virus discovery 
Novel Amalgaviruses of Rubber Dandelion

\section{Introduction}

Natural rubber is an essential material to the manufacture of 50,000 different rubber and latex products. A steadily increasing demand cannot be met only by the industrial exploitation of the rubber tree (Hevea brasiliensis). Viable alternative crops that could be established may supplement the demand, with carbon footprint savings, which is currently supported by diverse synthetic rubbers (Cornish, 2017). Rubber dandelion (Taraxacum kok-saghyz) is currently being developed as a sustainable source of natural rubber.

Thus, a robust metabolomic, genomic, and transcriptomic characterization should advance in parallel to explore the biological landscape of this important natural resource (Zhang et al., 2017). An additional aspect of this process is the exploration of potential microbes that could be associated with rubber dandelion. There are studies reporting the incidence of a virus in the related common dandelion $(T$. officinale), the Dandelion yellow mosaic virus (Secoviridae; Sequivirus - Bos et al., 1983). In addition, $T$. officinale has been described to be a reservoir host of important viral disease agents such as Tomato ringspot virus (Secoviridae; Nepovirus - Mountain et al., 1983), and Tomato spotted wilt orthotospovirus (Tospoviridae; Orthotospovirus - Groves et al., 2002). Interestingly, there are no reports describing viruses associated with rubber dandelion. Several members of a relatively new family of plant viruses have been identified in the last decade (Sabanadzovic et al., 2009). Amalgaviridae viruses are conformed by a dsRNA genome, containing two overlapping ORFs and an apparent common evolutionary history. Amalgaviruses are persistent, appear to be cryptic, share the genome architecture of Totiviridae and gene evolutionary resemblance to Partitiviridae (Martin et al., 2011). In this report, we present the characterization of two novel tentative members of the Amalgaviridae family and the Amalgavirus genus, the first viruses associated to T. kok-saghyz.

\section{Materials and Methods}

The first T. kok-saghyz transcriptome and associated reads as described by (Luo et al., 2017) were used as input for virus discovery. This transcriptome was produced from total RNA extracted from of 6 month old root samples of T. kok-saghyz of individuals of 6 different genotypes characterized by different rubber yields at The Ohio State University, and sequenced by Illumina Hiseq2000, obtaining 357,694,286 paired-end reads 100bp reads (GenBank accession numbers: genotype TK6 (SRR5181667); TK9 (SRR5181665); $\quad$ TK10 (SRR5181664); $\quad$ TK14 $\quad$ (SRR5181663); $\quad$ TK18 $\quad$ (SRR5181662); $\quad$ TK21 (SRR5181661)). The sequenced reads were quality evaluated using the FASTX-Toolkit, with a cut-off 
Novel Amalgaviruses of Rubber Dandelion

score of 30 (-q). The filtered reads then went through Trinity de novo assembly (version 2.2.0) using standard parameters. The filtered and normalized assembly (NCBI Transcriptome Shotgun Assembly (TSA) accession number GFJE00000000) of 55,532 transcripts was assessed by bulk searches using as query the complete virus refseq database available at ftp://ftp.ncbi.nlm.nih.gov/refseq/release/viral/ in a local server. BLASTX with an expected value of 10e-5 was employed as threshold, and hits were explored by hand. Tentative virus contigs were curated by iterative mapping of reads using Bowtie2 http://bowtie-bio.sourceforge.net/bowtie2/index.shtml virus Fragments Per Kilobase of transcript per Million mapped reads (FPKM) were estimated with Cufflinks 2.2.1 http://cole-trapnelllab.github.io/cufflinks/releases/v2.2.1/. Virus ORFs were predicted by ORFfinder https://www.ncbi.nlm.nih.gov/orffinder/ translated gene products were assessed by InterPro https://www.ebi.ac.uk/interpro/search/sequence-search and NCBI Conserved domain database v3.16 https://www.ncbi.nlm.nih.gov/Structure/cdd/wrpsb.cgi to predict domain presence and architecture. The 3D structure of the putative coat proteins was determined with the EMBOSS 6.5.7 Tool Garnier http://www.bioinformatics.nl/cgi-bin/emboss/garnier and coiled coil regions were predicted with COILS https://embnet.vital-it.ch/software/COILS_form.html using a MTIDK matrix. Predicted protein similarity plots were generated with Circoletto http://tools.bat.infspire.org/circoletto/ setting as E-value 10e-10. Phylogenetic insights based in predicted virus proteins were generated by MAFTT 7 https://mafft.cbrc.jp/alignment/software/ multiple amino acid alignments and FastTree 2.1.5 maximum likelihood phylogenetic trees computing local support values with the Shimodaira-Hasegawa test http://www.microbesonline.org/fasttree/. The FreeBayes v0.9.18S tool with standard parameters was employed for SNPs prediction https://github.com/ekg/freebayes. Results were integrated and visualized in the Geneious 8.1.9 platform (Biomatters Ltd.).

\section{Results}

The first publically-available RNA-Seq based T. kok-saghyz transcriptome, which was developed from pools of roots of genotypes with high and low rubber yields (Luo et al., 2017), was subjected to bulk BLASTX-NCBI searches using as query the complete virus refseq database. Interestingly, two transcripts presented consistent sequence identity to the Amalgavirus Southern tomato virus (Sabanadzovic et al., 2009) $(50 \%$ identity at the aa level; E-value $=0.0)$ and Blueberry latent virus $(49 \%$ identity at the aa level; E-value $=0.0$ ). The corresponding transcripts were curated by iterative mapping of RNA reads, 
Novel Amalgaviruses of Rubber Dandelion

which gave a mean coverage support of $49.1 \mathrm{X}$ and $77.7 \mathrm{X}$, respectively. The curated 3,409 nt and 3,413 nt long sequences were further explored in detail and designated tentatively isolate $\mathrm{OH}$ of rubber dandelion latent virus 1 \& 2 (RdLV1 \& RdLV2).

The RdLV1 determined genome presents a 143 nt 5UTR, a 97 nt 3UTR, and two partially overlapping ORFs on the positive strand (Figure 1.A). The predicted ORF1 encodes a 387 aa putative Coat protein (CP). The overlapping ORF2 encodes an 825 aa RdRP with a corresponding RNA_dep_RNAP domain (Pfam: pfam00680, E-value $=1.30 \mathrm{e}-07)$ at the 360-544 aa coordinates. Genome position 981 (_\$) presents a putative "slippery" sequence of the form ACU_UUU_CGC suggesting a host ribosomal +1 frameshift signal that could induce the generation of a characteristic 1,055 aa, $120 \mathrm{kDa}$ fusion protein (Figure 1.B). This slippery sequence is identical to the reported frameshifting signal of the Amalgavirus Rhododendron virus A (Sabanadzovic et al., 2010). The RdLV2 genome presents a $171 \mathrm{nt} 5$ UTR and a 100 nt 3 UTR (Figure 1.A). The predicted ORF1 encodes a 377 aa putative CP. The overlapping ORF2 encodes a 749 aa RdRP with a RNA_dep_RNAP domain (pfam00680, E-value $=2.99 \mathrm{e}-10$ ) at the 304 473 aa coordinates. Genome position 946 (_\$) presents a putative "slippery" sequence CAG_UUU_CGU that could induce the generation of a 1,046 aa, $118 \mathrm{kDa}$ fusion protein (Figure 1.B). The UTR regions of RdLV1 \& RdLV2 were A+U rich, as described for amalgaviruses (Sabanadzovic et al., 2009), ranging from $53.1 \%$ in the RdLV1 5 UTR to $61 \%$ in the 3UTR of RdLV2. The putative CP of RdLV1 \& RdLV2 were subjected to 3D structure prediction with the EMBOSS 6.5.7 Tool Garnier and coiled coil determination by COILS with a MTIDK matrix. A comparison of these predictions to that of reported Amalgavirus members (Figure 1.C) suggests that RdLV1 \& RdLV2 present a typical $\alpha$-helical central region with high probability of coiled coil as part of its tertiary structure, as is prevalent in Amalgaviridae (Nibert et al., 2016). It is worth mentioning that the predicted forms of potential slippery sequences of RdLV1 \& RdLV2 are of the general form UUU_CGN, similar to the experimentally validated sequence of influenza A virus (Firth et al., 2012). Theoretically, the ribosome may stall on a slippery sequence, making a pause at a rare codon ( pause may lead to a movement forward of one nucleotide. Translation resolves on the advanced ribosome in the +1 frame (Figure 1.B). This phenomenon has been predicted to be widespread among most plant amalgaviruses (Nibert et al., 2016). RdLV1 \& RdLV2 share a $55.9 \%$ genome nt identity and a $49.5 \%$ aa pairwise identity between their predicted RdRPs. Their proposed assignment as separate species is consistent with the species demarcation criteria for the genus Amalgavirus proposed by the International 
Novel Amalgaviruses of Rubber Dandelion

Committee on Taxonomy of Viruses (ICTV), which specifies an amino acid sequence divergence of over $25 \%$ at the RdRPs. The structural highlights of RdLV1 \& RdLV2 were compared to the ICTV recognized Amalgavirus species (Table 1). The predicted genome lengths and architectures, ORFs, UTRs, gene products, protein sizes, and general viral sequence cues are consistent with the proposed assignment of RdLV1 \& RdLV2 to the Amalgavirus genus. The predicted RdRP of RdLV1 \& RdLV2 were employed to glimpse some evolutionary insights of the identified viruses. Maximum-likelihood phylogenetic trees of RdLV1 \& RdLV2, and reported amalgaviruses, in the context of related viral families were generated, based on MAFTT protein alignments. The resulting trees evidently place RdLV1 \& RdLV2 in a cluster of amalgaviruses, and more distantly associated to new unclassified viruses and members of the Partitiviridae and Totiviridae families (Figure 2.A). The complete fusion protein (FP) of RdLV1 \& RdLV2 was explored in sequence similarity among recognized Amalgavirus species (Figure 2.B), and with closely related species (Figure 2.C) using the Circoletto tool (Darzentas, 2010), highlighting a stronger and broader link among the FP of RdLV1 \& RdLV2 and reported amalgaviruses. Interestingly, sequence identity robustly falls beyond the Amalgavirus genus. Further similarity with a species proposed to be a member of a new genus of fungi derived Amalgaviridae, the Zygosaccharomyces bailii virus $Z$ (proposed genus Zibavirus - ZbvZ) (Depierreux et al., 2016), is consistently low, supporting that both RdLV1 \& RdLV2 could be members of the Amalgavirus genus.

To confirm the presence of the identified viruses and explore their preliminary prevalence, we investigated six independent root total RNA samples of T. kok-saghyz which were individually sequenced by Illumina Hiseq2000 generating over 291 million 100 bp pair end reads, ranging between 5.2 Gb to 6.7 Gb per sample. Interestingly, the presence of the cognate viruses was confirmed in five of the six samples by iterative mapping of sequencing reads to the reference transcripts of RdLV1 \& RdLV2 (Figure 2.D). Virus relative RNA levels varied among samples, ranging from 3.69 FPKM for RdLV1 in TK-R14, to 12.11 FPKM for RdLV2 in TK-R6. In addition, in the TK-R18 sample, only RdLV2 was found, and both viruses were absent in TK-R21, suggesting that RdLV presence is dynamic and that mixed infections, whilst common, are not necessary. De novo assembly of the raw RNA data and further identification of RdLV isolates on the diverse samples were carried out in order to address preliminary virus diversity. Sequence variants among samples were reduced, presenting a high degree of homogeneity. Overall identity among individuals ranged from $98.3 \%$ to $99.4 \%$, which was roughly equivalent to the observed intra-individual identity which ranged between $99.2 \%$ and $99.5 \%$. A consistent identity among isolates 
Novel Amalgaviruses of Rubber Dandelion

was reported for Blueberry latent virus, when 35 diverse cultivars were assessed and over 99\% sequence identity among isolates was observed (Martin et al., 2011). Additionally, SNP were predicted by implementing the FreeBayes tool (Garrinson \& Marth, 2012), and 259 variants were identified among the CDS of RdLV1 \& RdLV2 (Figure 2.E); $78.37 \%$ of the polymorphisms involved the $3^{\text {rd }}$ position of the predicted codon, suggesting a tentative constraint to avoid amino acid changes and thus maintain structure and functional domains of the respective viruses.

\section{Discussion}

Recurrent attempts to transmit Amalgavirus via grafting and mechanical inoculation have failed. In addition, Amalgavirus are very efficiently transmitted vertically via seed (70-90\%), and have been associated with symptomless infections in their respective hosts (Sabanadzovic et al., 2010). The latter is consistent with our observations on tested rubber dandelions, which could not be linked with symptoms or altered phenotypes. Future studies should explore whether RdLV1 \& RdLV2 share the biological properties of persistence and exclude potential horizontal transmission. To our knowledge, there are no reports of interspecific transmission of amalgaviruses, and transmission by potential vectors has not been conclusively ruled out. Even though there are only four species of Amalgavirus species recognized by the ICTV, recent reports suggest that the diversity of this family of viruses is much more complex and widespread among plants (Nibert et al., 2016). The discovery of potentially cryptic viruses has been hampered by the targeted study of symptomatic organisms which lead to the biased discovery of pathogenic viruses (Geoghegan \& Holmes, 2017). Next generation sequencing is unraveling a new multifaceted virosphere paradigm, were viruses are widespread and associated to every organism (Greninger, 2017). The identified RdLV1 \& RdLV2 correspond to the first viruses associated with T. koksaghyz. The molecular characterization of these prospective members of the Amalgaviridae family is a first step on the path to advance the understanding of the intriguing biology of these potential endophytes and their economically important plant host.

-Nucleotide sequence accession number: The genome sequences of Rubber dandelion latent virus $1 \& 2$ have been deposited in NCBI GenBank under accession no MF197380 and MF197379

\section{Acknowledgments}


Novel Amalgaviruses of Rubber Dandelion

This work was supported by the Center for Applied Plant Sciences (CAPS), and the College of Food Agricultural and Environmental Sciences, The Ohio State University, and USDA National Institute of Food and Agriculture (Hatch project 230837).

\section{References}

Bos L, Huijberts N, Huttinga H, Maat D.Z. (1983) Further characterization of dandelion yellow mosaic virus from lettuce and dandelion. European Journal of Plant Pathology, 89, 207-222.

Cornish K. (2017) Alternative Natural Rubber Crops: Why Should We Care? Technology Innovation, 18, 244-255.

Darzentas N. (2010) Circoletto: visualizing sequence similarity with Circos. Bioinformatics, 26, 26202621.

Depierreux D., Vong M., Nibert M.L. (2016) Nucleotide sequence of Zygosaccharomyces bailii virus Z: evidence for +1 programmed ribosomal frameshifting and for assignment to family Amalgaviridae. Virus Research, 217, 115-124.

Firth A.E., Jagger B.W., Wise H.M., Nelson C.C., Parsawar K., Wills N.M., Napthine S., Taubenberger J.K., Digard P., Atkins J.F. (2012) Ribosomal frameshifting used in influenza A virus expression occurs within the sequence UCC_UUU_CGU and is in the +1 direction. Open Biology, 2, 120109.

Garrison E., Marth G. (2012) Haplotype-based variant detection from short-read sequencing. arXiv preprint, 1207, 3907.

Geoghegan J.L., Holmes E.C. (2017) Predicting virus emergence amid evolutionary noise. Open biology, 7, 170189.

Greninger A.L. (2017) A decade of RNA virus metagenomics is (not) enough. Virus Research, doi: 10.1016/j.virusres.2017.10.014

Groves R.L., Walgenbach J.F., Moyer J.W., Kennedy G.G. (2002) The role of weed hosts and tobacco thrips, Frankliniella fusca, in the epidemiology of Tomato spotted wilt virus. Plant Disease, 86, 573-582.

Luo Z., Iaffaldano B.J., Zhuang X., Fresnedo-Ramírez J., Cornish, K. (2017) Analysis of the first Taraxacum kok-saghyz transcriptome reveals potential rubber yield related SNPs. Scientific Reports, 7, 9939.

Martin R.R., Zhou J., Tzanetakis I.E. (2011) Blueberry latent virus: an amalgam of the Partitiviridae and Totiviridae. Virus Research, 155, 175-180. 
Novel Amalgaviruses of Rubber Dandelion

Mountain W.L., Powell C.A., Forer L.B., Stouffer R.F. (1983) Transmission of tomato ringspot virus from dandelion via seed and dagger nematodes. Plant disease, 67, 867-868.

Sabanadzovic S., Ghanem-Sabanadzovic N.A., Valverde R.A. (2010) A novel monopartite dsRNA virus from rhododendron. Archives of Virology, 155, 1859-1863.

Sabanadzovic S., Valverde R.A., Brown J.K., Martin R.R., Tzanetakis I.E. (2009) Southern tomato virus: the link between the families Totiviridae and Partitiviridae. Virus Research, 140, 130-137.

Nibert M.L., Pyle J.D., Firth A.E. (2016) A +1 ribosomal frameshifting motif prevalent among plant amalgaviruses. Virology, 498, 201-208.

Zhang Y., Iaffaldano B.J., Zhuang X., Cardina J., Cornish K. (2017) Chloroplast genome resources and molecular markers differentiate rubber dandelion species from weedy relatives. BMC Plant Biology, 17, 34. 
Novel Amalgaviruses of Rubber Dandelion

Table 1 Diverse structural highlights of RdLV 1 \& 2 in comparison with ICTV recognized Amalgaviridae species. GS: Genome size (nt), 5'U: 5'UTR length (nt), OR1: ORF 1 length (nt), OR: Overlapping region (nt), OR2: ORF 2 length (nt), 3U: 3UTR length (nt), SLPs: Slippery sequence, SLPp: SLP position, FP: Fusion protein length (aa), RP: RdRP (aa), CP: Putative Coat protein length (aa), RPi, CPi, GSi: RdRP, CP, and complete genome sequence identity of the corresponding Amalgavirus in relation to RdLV1.

\section{Figure Legends}

Figure 1. Molecular characterization of RdLV1 \& RdLV2 (A) Rubber dandelion latent virus 1 \& 2 (RdLV1 \& RdLV2) linear monopartite dsRNA genome are 3,409 \& 3,413 nt long, arranging a translation strategy based in two partially overlapping ORFs. The RdLV1 genome presents a 143 nt 5'UTR and a 97 nt 3 UTR. The predicted ORF1 encodes a 387 aa putative Coat protein. The overlapping ORF2 encodes a 825 aa RNA dependent RNA Polymerase. Genome position 981 (_\$) presents a putative "slippery" sequence that could induce the generation of a $120 \mathrm{kDa}$ fusion protein. The RdLV2 genome presents a $171 \mathrm{nt}$ 5 UTR and a $97 \mathrm{nt}$ 3 UTR. The predicted ORF1 encodes a 377 aa putative Coat protein. The overlapping ORF2 encodes a 749 aa RdRP. Genome position 946 (_\$) presents a putative "slippery" sequence that could induce the generation of a $118 \mathrm{kDa}$ FP. (B) Potential programmed ribosomal frameshifting of RdLV1 \& 2. The RdLV1 ACU_UUU_CGC motif and RdLV2 CAG_UUU_CGU motif, of the general form UUU_CGN, are +1 ribosomal frameshifting motif prevalent among most plant amalgaviruses. (C) 3D structure prediction of the corresponding Coat proteins of RdLV1 \& 2 and of reported amalgaviruses, assessed with the EMBOSS 6.5.7 tool Garnier represented on top, and coiled coil determination by COILS with a MTIDK matrix as a line graphs. Regions of high coiled coil probability are constrained to the typical $\alpha$-helical central region of the CPs.

Figure 2. Phylogenetic insights and exploratory prevalence of RdLV1 \& RdLV2 (A) Maximumlikelihood phylogenic tree of the RdRP predicted protein of reported amalgaviruses in the context of related viral families based on a MAFTT multiple alignments. Numbers at the nodes indicate percentage of bootstrap consensus support values obtained for 1000 replicates. Sequence similarity levels of 
Novel Amalgaviruses of Rubber Dandelion

amalgaviruses Fusion Proteins among the Amalgavirus genus (B) and RdRP proteins of related viruses (C) expressed as Circoletto diagrams. FPs or RdRPs are depicted clockwise, and sequence similarity is visualized from blue to red ribbons representing low-to-high sequence identity. (D) Virus RNA levels expressed as FPKM of NGS sequenced rubber dandelion total RNA root samples. Values for RdLV1 are depicted in blue columns and values for RdLV2 in orange columns. (E) RNAseq based read mapping graphs of RdLV1 and RdLV2 with the 6 combined RNA libraries. Tracks from top to bottom represent coverage per base, sequence identity from red to green (higher), and SNP prediction. GenBank accession numbers and abbreviations for the respective viruses are Southern tomato virus (STV, NC_011591), Rhododendron virus A (RV-A, NC_014481), Blueberry latent virus (BBLV, NC_014593), Vicia cryptic virus M (VCV-M, EU371896), Hubei partiti-like virus 59 (Hplv, APG78262), Beihai barnacle virus 14 (Bbv14, APG78182), Zygosaccharomyces bailii virus Z (ZbvZ, KU200450), Colletotrichum higginsianum dsRNA virus 1 (Chv1, NC_028242), Heterobasidion partitivirus P (HpP, AAK52739), Radish partitivirus (AY748911), Vicia cryptic virus (VCV, EF173396), Saccharomyces cerevisiae virus L-A (ScV-LA, NC_003745), Penicillium stoloniferum virus S (PsvS, NC_007539), Aspergillus ochraceous virus (AoV, EU118277), Cryptosporidium parvum virus 1 (CpV1, CPU95995), Pepper cryptic virus 1 (PCV1, JN117276), Trichomonas vaginalis virus (TvV, NC_003824), Fig cryptic virus (FCV, NC_015494), Atkinsonella hypoxylon virus (NP_604475). $\alpha$ : Alphapartitivirus genus, $\beta$ : Betapartitivirus genus, $\gamma$ : Gammapartitivirus genus, $\delta$ : Deltapartitivirus genus, C: Cryspovirus genus. 


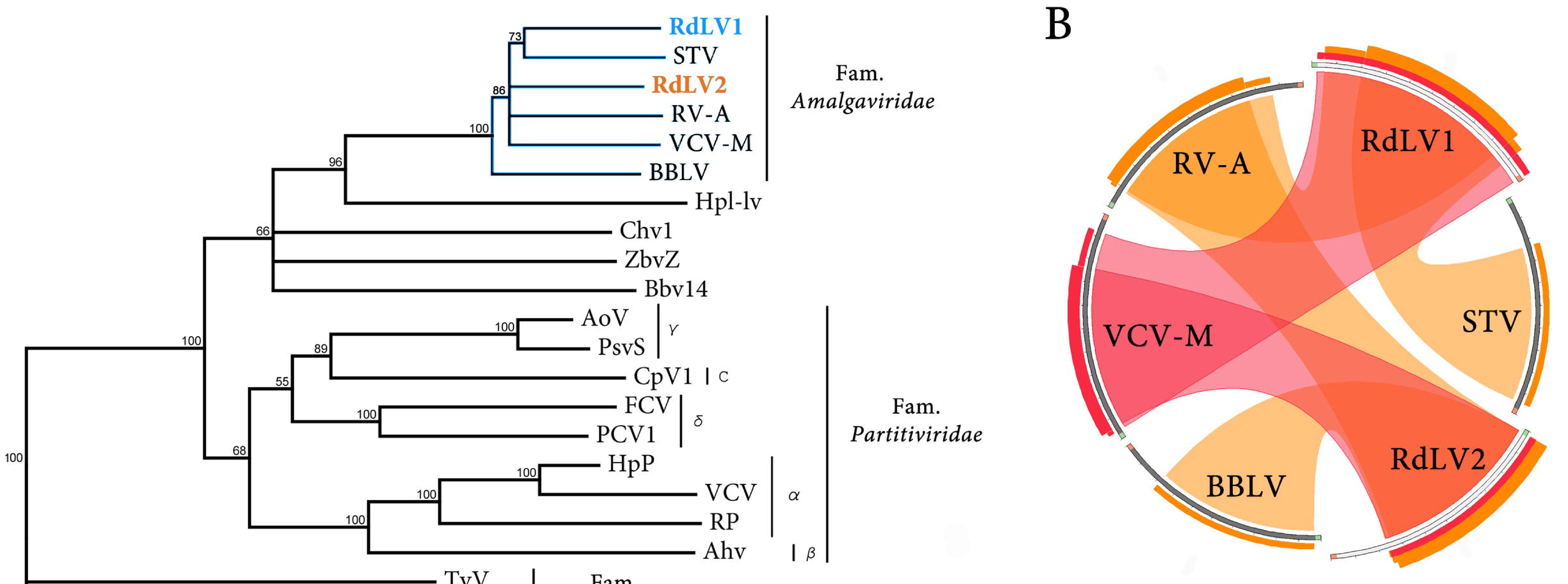

E

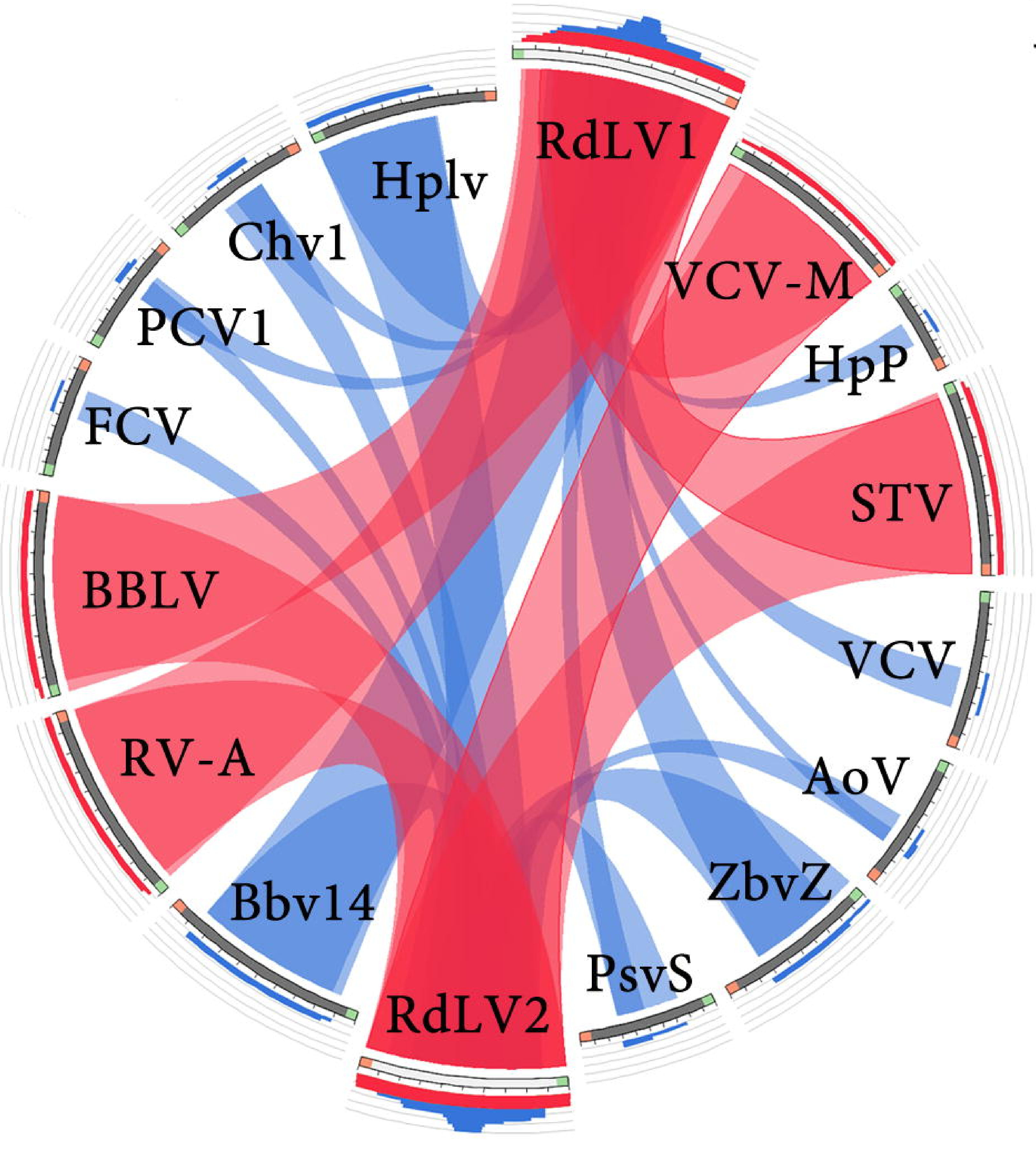

D

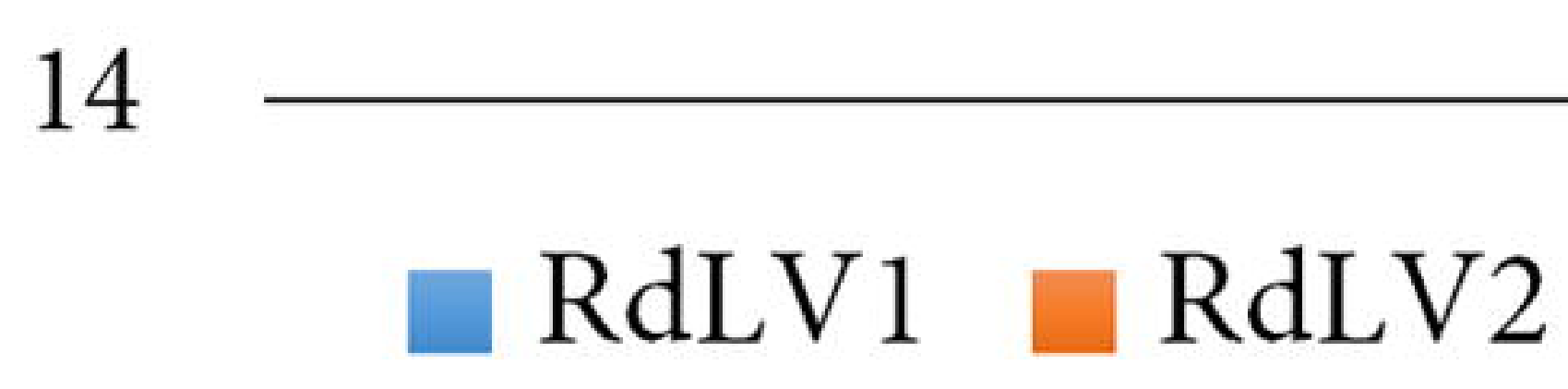

12

10
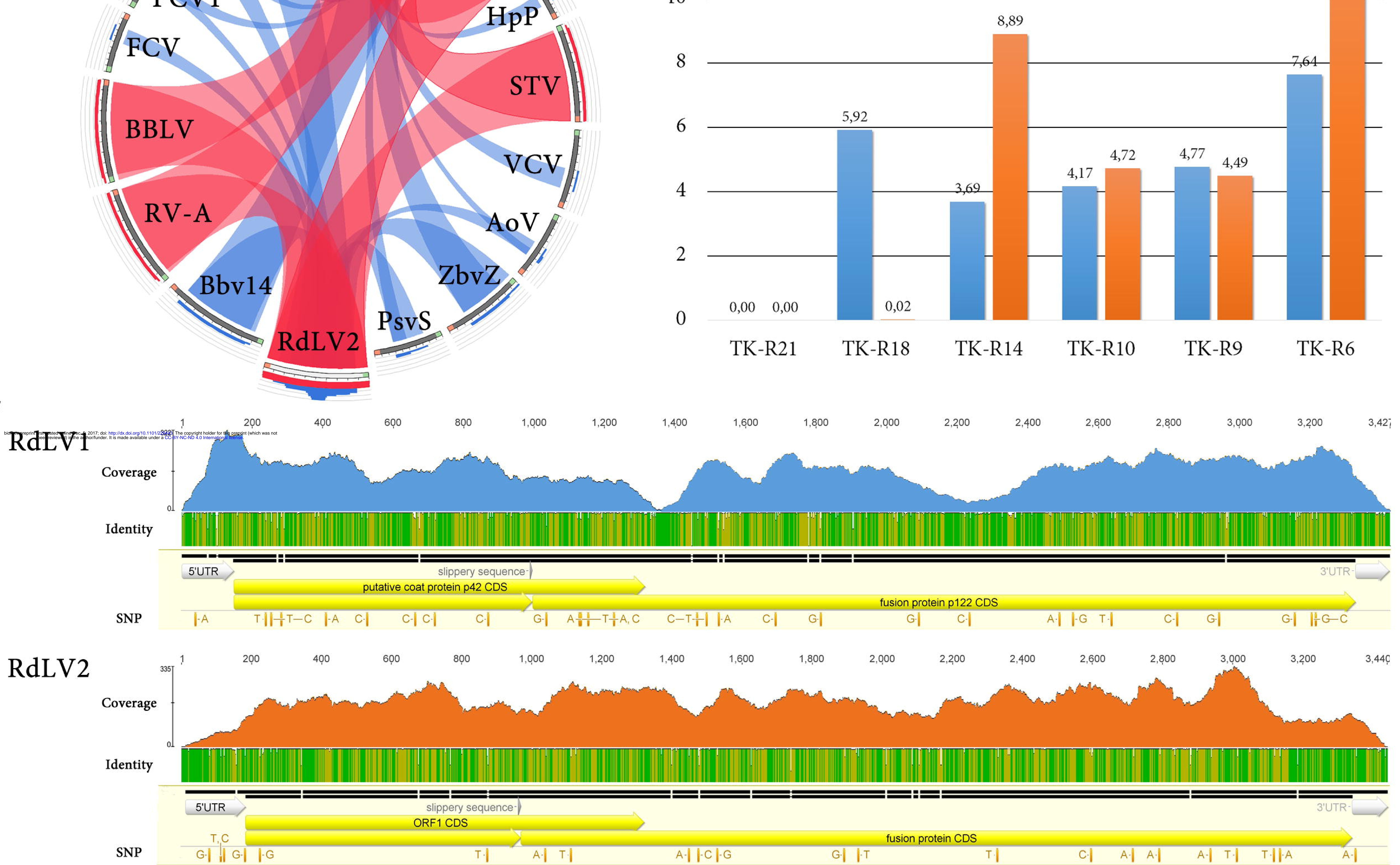


\section{Molecular Identification and Characterization of Two Rubber Dandelion Amalgaviruses}

Humberto Debat, Zinan Luo, Brian J. Iaffaldano, Xiaofeng Zhuang and Katrina Cornish

\begin{tabular}{|c|c|c|c|c|c|c|c|c|c|c|c|c|c|c|c|}
\hline Amalgavirus & GS & $5^{\prime} \mathbf{U}$ & OR1 & OR & OR2 & $3^{\prime} \mathbf{U}$ & SLPs & SLPp & FP & $\mathbf{R P}$ & $\mathbf{C P}$ & $\mathbf{R P i}$ & $\mathbf{C P i}$ & GSi & Accession n. \\
\hline $\begin{array}{l}\text { Rubber dandelion latent virus } \\
1\end{array}$ & 3,409 & 143 & 1,164 & 473 & 2,575 & 97 & ACU_UUU_CGC & 981 & 1,055 & 825 & 387 & $\begin{array}{l}----- \\
\end{array}$ & $\begin{array}{l}---- \\
\end{array}$ & $\begin{array}{l}---- \\
\end{array}$ & MF197380 \\
\hline $\begin{array}{l}\text { Rubber dandelion latent virus } \\
2\end{array}$ & 3,413 & 171 & 1,134 & 242 & 2,250 & 100 & CAG_UUU_CGU & 946 & 1,046 & 749 & 377 & 49.5 & 21.5 & 55.9 & MF197379 \\
\hline Southern tomato virus & 3,437 & 137 & 1,134 & 233 & 2,289 & 110 & CUU_AGG_CGU & 983 & 1,063 & 763 & 378 & 49.5 & 22.0 & 55.7 & NC_011591 \\
\hline Blueberry latent virus & 3,431 & 166 & 1,128 & 359 & 2,397 & 99 & UCU_UUU_CGU & 979 & 1,055 & 799 & 376 & 46.2 & 19.5 & 54.8 & NC_014593 \\
\hline Rhododendron virus $A$ & 3,427 & 94 & 1,215 & 405 & 2,424 & 47 & ACU_UUU_CGC & 1,181 & 1,078 & 808 & 405 & 48.0 & 22.1 & 54.4 & NC_014481 \\
\hline Vicia cryptic virus $M$ & 3,434 & 142 & 1,185 & 287 & 2,277 & 117 & ACU_UUU_CGU & 1,089 & 1,058 & 759 & 395 & 47.3 & 19.7 & 54.8 & EU371896 \\
\hline
\end{tabular}

Table 1 Diverse structural highlights of RdLV 1 \& 2 in comparison with ICTV recognized Amalgaviridae species. GS: Genome size (nt), 5U: 5UTR length (nt), OR1: ORF 1 length (nt), OR: Overlapping region (nt), OR2: ORF 2 length (nt), 3U: 3 UTR length (nt), SLPs: Slippery sequence, SLPp: SLP position, FP: Fusion protein length (aa), RP: RdRP (aa), CP: Putative Coat protein length (aa), RPi, CPi, GSi: RdRP, CP, and complete genome sequence identity of the corresponding Amalgavirus in relation to RdLV1. 